METASCIENCE AS A SCIENTIFIC SOCIAL MOVEMENT

David Peterson

Aaron Panofsky

UCLA 


\section{METASCIENCE AS A SCIENTIFIC SOCIAL MOVEMENT}

For four days in early September 2019, hundreds of scholars congregated at Stanford University for an academic symposium. Forty presenters and panelists shared research at various stages of completion before adjourning to network and gossip at coffee klatches and bars. It was, in many respects, a normal academic event. Yet, some features of this symposium stuck out. For one, it brought together an unusual mix of people. Psychologists and physicists, data scientists and philosophers, biologists and economists shared the stage. Secondly, although the event was ostensibly an opportunity to share research, attendees at the conference included many nonscientists including representatives from the NSF, DARPA, the Sloan Foundation, and Wellcome Trust; Silicon Valley firms like Facebook and Y Combinator; and science reporters.

The symposium was entitled "Metascience: The Emerging Field of Research on the Scientific Process" and represented a coming out party for an area of science that is having a moment. Emerging out of the "reproducibility crisis" in science, metascientists have become central players in debates about research integrity, scholarly communication, and science policy. Beyond its role diagnosing and treating the reproducibility crisis, metascience has offered tantalizing glimpses of a radically reimagined science, a world where the journal system is replaced with something modular and nimble and where vast pools of freely available data are reconfigured and reused in currently unthinkable ways. The scale of their project and the ambition of their vision has captured the imagination of journalists and funders.

During the same days as the Metascience Symposium, twenty-two hundred miles away, another group of science studies scholars was sitting in a conference room in New Orleans discussing the same topics. The Society for the Social Studies of Science hosted three consecutive panels on the replication crisis and its implications. What is remarkable about these two events is how little visibility there is between the groups given the enormous overlap in subject matter.

The goal of this article is to introduce metascience to STS scholars, detail the scientific ideology that is apparent in its articles, strategy statements, and research projects, and discuss its institutional and intellectual future. Put simply, metascience is a scientific social movement that seeks to use the tools of science- especially, quantification and experimentation- to diagnose problems in research practice and improve efficiency. It draws together data scientists, 
experimental and statistical methodologists, and open science activists into a project with both intellectual and policy dimensions. Intellectually, metascience produces quantitative studies meant to describe and evaluate science on a macro scale. Metascientists then use those studies to motivate reforms in scientific practice including more elaborate reporting requirements, data deposition demands, and more replications.

This is a worthy object of study for several reasons. Metascientists have been remarkably successful at winning grants, motivating news coverage, and changing policies at science agencies, journals, and universities. Even scientists who are unaware of metascience have seen anxious stories about replication failures and confront new bureaucratic demands; metascientists have both called attention to these problems and situated themselves as key players in their solution. Moreover, metascience represents the apotheosis of several trends in research practice, scientific communication, and science governance. These include increased attention to methodological and statistical criticism of scientific practice (Freese \& Peterson 2018; Nelson 2020), the promotion of "open science" by science funders and journals (Mirowski 2018), the growing importance of both preprint and data repositories for scientific communication (Bohlin 2004; Leonelli 2016), and the new prominence of data scientists as research makes a turn toward Big Science (Arribas-Ayllon et al. 2019). Although this previous work motivates and informs our argument, our work focuses on the largely unacknowledged interaction of these forces and the emergent ways they're combining to change scientific practice a systemic scale.

The reproducibility crisis emerged at the same time as other institutional crises in media and government. The debates that often follow these crises polarize into those advocating a return to core values and those who argue the crisis demands radical social transformation. Metascience, oddly, does both. Like organizations that provide media scorecards or that advocate for increased transparency in government, metascience represents a modernist solution to a modernist problem, an intensification of already preexisting values of openness, accountability, and quantification. Yet, through this embrace of the values in which science has historically been cloaked, metascience seeks to create new systems that will lead science into an unprecedented future.

We have barely begun confronting the post truth moment and understanding how it relates to longstanding issues of science, expertise, and democracy. The crisscrossing lines of causes and ramifications can seem overwhelming and nonsensical. Thus, it behooves us to pay 
special attention to those inflection points where multiple streams flow together and gain force. The metascience symposium was such a moment.

\section{DATA SOURCES}

The Metascience Symposium is a touchstone for us throughout this article, and is useful for framing purposes because of its specificity. Topics of interest to metascience like research methodology, meta-analysis, and scientific communication are featured in some form at most disciplinary conferences. However, the goal of the symposium was explicitly, as one of the organizers said in his opening address, to "break those boundaries that are largely limited by language in making connections across our disciplinary domains." That is, the goal was to demonstrate the transdisciplinary nature of their project and facilitate communication across silos. Because this was one of the first events to physically bring together this heterogeneous group of scholars, the presentations mixed the empirical and programmatic, providing a sense of, both, what metascience is and what it is not.

Of the 30 scheduled talks, 29 were recorded and uploaded onto the conference website. In addition to the presenter talks, there were three panels — one for funders, one for journalists, and one for "skeptics." We watched the 32 available talks. The list of participants provided a list of interviewee subjects. We interviewed 22 people who attended the symposium, including three of its organizers (Brian Nosek, Jonathan Schooler, and Jan Walleczek) and the president of the philanthropic organization that provided its funding (Bruce Fetzer of the Franklin Fetzer Fund).

We interviewed an additional 36 people involved in the various strands of metascience. This included two editors of Science; representatives from the NSF, Moore Foundation, Arnold Ventures, and the Sloan Foundation; and the leaders of organizations that promote open science and manage open access repositories. ${ }^{1}$ The 58 interviews averaged XX minutes.

Finally, we read extensively in the published articles written by metascientists and the white papers produced by metascientific and open science organizations.

\footnotetext{
1 Because many of the groups are known by acronyms, the following is a reference list:

COAR- Confederation of Open Access Repositories

Cos- Center for Open Science

DARPA- Defense Advanced Research Projects Agency

METRICS- Meta-research Innovation Center at Stanford

SoS- Science of science

SPARC- Scholarly Publishing and Academic Resources Coalition
} 


\section{METASCIENCE AS A SOCIAL MOVEMENT}

Although we argue that metascience is a movement that arose in the past 15 years, the term metascience is nearly a century old and has been used to describe variety of activities that are only loosely connected to the current movement. In one of the earliest uses, the logical positivist philosopher Charles Morris (1946) suggested that a semiotically-grounded "metascience" could be used to provide a unifying structure to empirical sciences that differed in their concrete practices. Later, the philosopher Mario Bunge (1959) used the term in a way that is synonymous with the philosophy of science (a tradition still visible in the journal Metascience which publishes book reviews for books in the philosophy of science and technology).

Modern metascience is neither a descendent of the philosophy of science nor the empirical research characteristic of the last four decades in the social studies of science. Instead, it is better understood as an intellectual and policy reaction to the popular narrative that science is currently undergoing a "reproducibility crisis." This is the belief that science faces widespread replication problems stemming from a mix of increased competition, incentives that reward improbable findings, and lax policing of bad or fraudulent science.

Metascience is what Frickel and Gross (2005) have referred to as a "scientific/intellectual movement" (SIM) which are "collective efforts to pursue research programs or projects for thought in the face of resistance from others in the scientific or intellectual community" (206). Integrating research in the sociology of ideas, STS, and social movements, they outline a general theory of SIMs. To quickly summarize, SIMs succeed when high-status actors articulate problems in current intellectual trends, frame a solution that is attractive to others, develop a common identity through interaction with other critics, and are able to secure resources to ensure the self-perpetuation of the movement's goals. What's come to be known as the "Cognitive Revolution," for example, emerged when psychologists, frustrated by the limitations of behaviorist dogma, articulated a new vision of psychological science rooted in an information processing metaphor of human cognition. Securing funding from the Alfred P. Sloan Foundation, they were able to attract a diverse group of psychologists, computer scientists, and linguists to symposia and collaborative projects, eventually establishing ongoing conferences, journals, and departments (Gardner 1987; Miller 2003). 
Rather than being an attempt by nonexperts to engage more directly with science (e.g., Brown et al. 2004; McCormick 2007), SIMS are instigated by experts themselves to transform their fields. Yet, metascience in an unusual SIM in several ways. First, rather than a disciplinary or even multi-disciplinary movement, metascience is truly transdisciplinary. Its aims are not limited to any particular domain. Rather, they seek reforms science-wide. Second, metascience is largely unconcerned with the content of science. It is not interested in the epistemic status of any specific facts or theories. Both its critiques about the reproducibility crisis and proposed reforms are pitched at a macro level. Thus, it is concerned with the general form of science-how it is practiced, recorded, stored, and shared. Third, because it claims authority over scientific practice writ large, it seeks to create another field of scientific activity which stands not shoulder to shoulder with the other disciplines in the crowded field of fields, but stands, in some sense, over them. Finally, rather than a strictly intellectual movement, metascience has both intellectual and policy dimensions. Thus, it represents what Epstein (2008:21) has referred to as a "hybrid coalition" in which experts, bureaucrats, and policymakers are drawn together. In this case, metascience represents the confluence and cross-fertilization of three, preexisting trends: (1) statisticians and experimental methodologists promoting greater research integrity, (2) quantitative studies of science by data scientists (what is called "science of science" or SoS), and (3) open science activism. These three groups found common cause and new possibilities for media attention, funding, intellectual prestige, and influence through both promoting the replication crisis and offering reforms.

In this section, we discuss the significance of the replication crisis narrative and the attractions it held for methodologists, data scientists, and open science activists.

\section{CRISIS DIAGNOISIS AND TREATMENT}

The history of metascience cannot be understood apart from the anxiety about a possible reproducibility crisis. Metascience has produced the tools that supposedly uncovered the crisis through statistical critiques, meta-analyses, and mass replications. Together, these provided the evidence that activists have used to demonstrate systemic problems across fields. And, metascientists have positioned themselves as key players in solving the crisis by pushing for interventions in everything from scientific training to reporting. 
The crisis narrative itself has old roots. Critiques of null hypothesis significance testing have been around for decades. Yet, Ioannidis's (2005) article "Why most published research findings are false" managed to capture attention in ways previous attempts had not. Although commentaries on statistical power and criticisms of p-values were common, the idea that poor statistical practice could produce entire areas of active research that were composed of noise had not been widely appreciated. Thus, it is only in the late 2000 s that the term "reproducibility crisis" begins to appear in the academic literature. Ioannidis's argument, which was largely based upon the statistical qualities of hypothesis testing was given added urgency when accusations and evidence of irreproducibility swept through various fields (Begley \& Ellis 2012; Gelman 2016; Prinz et al. 2011). More ominously, increased attention was given to cases of research misconduct. In 2010, the blog Retraction Watch was founded and was the first forum built to publicize and keep track of retractions. The blog subjected a process that typically played out in the shadows to the harsh spotlight of the internet and suggested that cases of fraud, manipulation, and plagiarism were far more common than previously suspected. Together, the statistical critiques, replication failures, and fraud scandals painted an ugly picture.

During this period, a diagnosis for problems in science began to crystallize (Freese \& Peterson 2018). Although there are several variations, the essence of the diagnosis is that the incentive system in science is misaligned and this skews science toward nonreproducible findings. Nosek and colleagues (2012) put it concisely: "The real problem is that the incentives for publishable results can be at odds with the incentives for accurate results" (616). In Merton's (1973) classic account, scientists were extraordinarily careful because every publication provided an opportunity for competitors to criticize and, potentially, mar an author's reputation. The evidence of the replication crisis, however, indicated that scientists no longer feared the scrutiny of their peers. Critics began to believe the benefits of major publication-jobs, promotions, grants, etc. - outweighed reputational risk and led scientists to overstate, manipulate, and, even, commit fraud with little fear of discovery. Worse, it was argued this behavior could become a vicious circle as young researchers learn that the only way to compete in science is to cut corners sinking entire fields into a mire of poor or fraudulent research (Smaldino \& McElreath 2016).

Within their crisis diagnoses, metascientists propagated an implicit psychology, sociology, and philosophy of science. Psychologically, they framed scientists as inherently, insurmountably biased. Even with the best of intentions, individual researchers will make 
decisions that bend results toward expected or favored outcomes. To counter this, they aimed to eliminate subjectivity when possible and, when not, force decisions to be made explicitly and transparently. Sociologically, they viewed scientists largely as economic actors, responding to incentives to maximize rewards. When the incentive system is organized in inefficient or harmful ways (say, by not encouraging replications), science suffers. To counter this, they worked to align the individual incentives of scientists for personal rewards in ways that benefit the whole community. Philosophically, the metascientific picture of science is that of an evolving datascape. Its central task is not the invention of theories nor the construction of experimental systems, but the creation, collection, curation, and analyses of data. Thus, it shifts emphasis away from polished publications and laboratories to data repositories. To power this, metascientists produce replications to increase the statistical power of datasets while working to make scientific data accessible and interoperable.

The holism and scale of the metascientific vision distinguishes the movement from previous moments of methodological debate and scandal. Typically, scandals in science are located in particular researchers or, perhaps, labs. What was novel about the reproducibility crisis is that its diagnosis is systemic. Thus, it is argued, a systemic response is required. Null hypothesis testing is a popular method across fields. Issues with small sample sizes and weak effects cross disciplines. Likewise, the incentive structure of science is shared. The drive for strong, novel findings is not unique to psychology or medicine. Everyone is implicated.

Metascience is about big-S Science, and its reforms are aimed at widespread, deep, and lasting transformations. The articles in this genre advocate for a lengthy list of reforms that cross nearly every aspect of scientific practice and communication. Three influential papers (Begley \& Ioannidis 2015, Munafò et al. 2017; Nosek \& Bar-Anan) offer series' of proposals including creating guidelines for best research practice, encouraging better reporting of methodological and analytical choices, improving methodological training, embracing the possibilities of digital communication (e.g., no more page limits), and a dozen other ideas encompassing nearly every aspect of research. Sprinkled between these largely uncontroversial suggestions is a set of goals whose meaning is more obscure, and point to the radical vision of change metascientists propose. These include encouraging ongoing mass replications, decoupling the publication process from the evaluation process through the use of preprint servers, transforming peer review into an open 
and potentially endless process, and making open data practices the norm to bolster research integrity and foster new possibilities of data reuse.

\section{THE CRISIS NARRATIVE}

Yet, the narrative of crisis must not be taken at face value. It is inarguably a significant actors' category that has already had profound impacts regarding science policy and practice, but is there actually a crisis? Rather than debate the merits of the case, here, we make a theoretical point. Like other crises in society, there is no objective answer to when a problem becomes a crisis. Instead, a crisis becomes a crisis through the work of actors who see a problem, diagnose it, and convey the threat as something terrible that requires urgent action.

Becker's (2008) classic work on moral crusades and moral entrepreneurs outlined how this works. Moral crusades are launched when a person or group feels that existing rules have failed to curb some evil. In the case of the replication crisis, metascientists argued that current research standards perverted scientific findings and allowed scientific investment to be wasted in fruitless efforts. For the crusader's message to carry, however, it needs to be amplified, and the replication crisis found two ready amplifiers. First, there were science journalists who took on the issue of bad science and become actors within this hybrid movement. Summarizing the critique above, the subtitle of one journalist's book promised to reveal "How Sloppy Science Creates Worthless Cures, Crushes Hope, and Wastes Billions" (Harris 2017). Writers at the New York Times, the Atlantic, Vox, Buzzfeed News, and other outlets produced a seemingly unbroken stream articles highlighting problems in science. ${ }^{2}$ Second, the emergence of Twitter as a medium for academic communication allowed scientists to share stories of problems and provided a way

\footnotetext{
2 The names of the articles and essays reveal both the scope and tone of the coverage:

- "The experiments are fascinating. But nobody can repeat them" (Gelman 2010)

- "The inevitable evolution of bad science: A simulation shows how the incentives of modern academia naturally select for weaker and less reliable results" (Yong 2016)

- "Cancer scientists are having trouble replicating groundbreaking research" (Belluz 2017)

- "These people are trying to fix a huge problem in science" (Chivers 2017)
} 
for scientific reformers to network with colleagues outside their disciplines, thus, creating a transdisciplinary (or, metascientific) dialogue. ${ }^{3}$

Moral crusades are initiated by moral entrepreneurs who seek to create and enforce new rules to stanch the spread of the evil they see. With a resonant message, moral entrepreneurs then seek to alter the system to root out the threat. According to Becker, there are "rule creators" who establish new laws, standards, and norms and "rule enforcers" who ensure that the new strictures are followed. As the crisis narrative gained traction, activists worked with funders, journals, and universities to change scientific practice and develop new rules and mechanisms to entice, coax, or coerce cooperation from scientists.

Through this explanation, we are not trying to minimize reproducibility problems or suggest that they are mere social constructions. However, we do mean to highlight the social work that goes into framing a problem as a crisis. Although will not explore the debate in depth, it is significant that nearly every aspect of the crisis diagnosis is contested: the idea that reproducibility problems are malignant, that they represent a crisis, and that they are sciencewide all find powerful critics. ${ }^{4}$ For the reproducibility crisis to become widely accepted as fact,

\footnotetext{
3 In our interview, Brian Nosek noted the role of social media: "I think Twitter actually has been a key part of fomenting an interdisciplinary cross talk that has facilitated a study of metascience that otherwise was very local, very small impact, and would die out very quickly." Likewise, another presenter at the Metascience symposium wrote to us saying, "A LOT of metascience happens on twitter." Frickel and Gross argue that SIMs are more likely to be successful when they have access to "micromobilization" contexts that allow participants to channel emotional energy. Prior to social media, scientists from different fields have few options to actually exchange views and reinforce each other in this way.

4 It is notable that much of the early evidence which metascientists used (and continue to use) as evidence of crisis has serious flaws. For instance, a survey in Nature suggesting widespread replication issues throughout science has been cited nearly 2,000 times (Baker 2016). Yet, the survey was based upon a convenience sample of scientists willing to take a 20-minute survey on the topic of reproducibility. Moreover, the article is in Nature's "News Feature" section rather than "Research." It is, thus, unclear, what sort of review it underwent. Similar issues for a survey of "questionable research practices" in psychology (John et al. 2012) led to a testy exchange in which Norbert Schwarz accused the authors of wording questions in a biased way to manufacture evidence of a crisis (the critiques were later published [Fiedler \& Schwarz 2016]). Finally, the Amgen study, which has been cited 2,300 times has been used as evidence of serious reproducibility problems in preclinical cancer research. However, the article did not disclose what articles it attempted to replicate, nor the processes they went through to try to replicate them, nor what they considered to be a success or failure. In the "critics" panel at the Metascience Symposium, NIH Senior Investigator Jon Yewdell blasted the study:
} 
metascientists had to craft a narrative that was convincing to enough to journalists, members of the lay public, fellow scientists, and policymakers to overcome these objections.

Thus, regardless of whether one believes there is a legitimate crisis of reproducibility or not, it must be acknowledged that the crisis narrative added significant tailwinds to metascience both intellectually and financially. The connection between the crisis narrative and metascience was clear in the introductory talk given by one of the organizers of the Metascience Symposium: "The exciting opportunity that we have here is to take the momentum that has been generated by this so-called 'reproducibility crisis' and use that to fuel the emergence of this new field of metascience." Although, as we will argue later, metascientists have generally downplayed the crisis narrative in their published work, it has provided new opportunities to the three groups that make up the burgeoning field.

\section{HOW THE CRISIS BROUGHT TOGETHER THE THREE STRANDS OF METASCIENCE}

The metascience movement formed as a reaction to the reproducibility crisis. Yet, it did not emerge from nothing. Rather, it found fertile soil at the intersection of open science activism, the science of science, and statistical and methodological critiques. Each of these domains has their own, largely independent history, but the replication crisis provided new opportunities for each and these opportunities were magnified through interaction with the others.

Open science activism. Before "open science," there was "open access," that was started largely by research librarians and their allies in the 1990s to democratize access to science and lower costs. They achieved some success launching open access journals and convincing journals to offer open access publication options. The reproducibility crisis opened up a new front in the battle for openness. In the popularized narrative of the reproducibility

\footnotetext{
That first paper that came out from the Amgen group, this was the very definition of chutzpah: 'Well, we can't replicate these studies, but we're not going to tell you what they were and we're not going to tell you how we did it.' That's meaningless. That's malpractice by the journal editor who published that. And that's one of the things that got the whole crisis going.

We are not taking sides in these debates, but they reveal the contentious nature of the crisis diagnosis.

5 "Open software" has also played a role in metascience, but mainly through the data science/science of science pathway. Specifically, the focus on the openness of code, decentralization, and modifiability of systems that one finds in metascience discussions (e.g., Nosek \& Bar-Anan 2012; Ross-Hellauer et al. 2019; Uhlman et al. 2019) can be traced to the utopian rhetoric of early open software activists (Kelty 2008).
} 
crisis, scientific problems are made and go uncorrected because the products of science are "closed" for inspection. Because researchers are rarely required to publicly post their data, code, or details of their analytic decisions, errors (unintentional or not) can slip through and fester. Thus, a key plank of their reform efforts involve encouraging and/or mandating open science. Activists who had been primarily working toward open access found themselves in an environment where a different dimension of "openness," open data, became a hot topic in science policy. Research librarians saw this as an opportunity to create a new role for themselves at the same time their traditional role, helping scholars find resources, had become threatened. Cornell librarian and former head of arXiv Oya Reiger, told us that "There's an ongoing tension, anxiety, especially over the last 10 years. Users discover content on the web now, whether it's Google Scholar or Research Gate. Very rarely they come to the library to find resources. I think libraries are feeling like their role in discovery is really weakening, and then there are all kinds of other services that they can provide, but they are trying to transition their workforce to assume new roles." Research librarians were able to leverage existing skills in collecting, preserving, organizing, and sharing information for a new set of responsibilities. They increasingly teach data management skills to research scientists and maintain the institutional repositories that were necessary to meet the demands that were beginning to be required by funding agencies and journals.

Science of science. The science of science can be sourced back to the physicist Derek de Solla Price, who conducted some of the earliest quantitative studies of science. This field of scientometrics that resulted has produced decades of work showing how science operates and how it changes over time. However, the past decade has seen an upsurge of interest in this field. In a recent article introducing the SoS, Fortunado and colleagues (2018) note that recent growth in the field is the result of two developments: (1) increases in data availability because of the migration of research journals and data online and (2) an influx of natural and computational scholars into the field. Migrants from physics, computational biology, and computer science began applying the tools of statistical mechanics to the massive datasets recently made available (datasets in SoS commonly contain hundreds of thousands or more articles). As Ioannidis and colleagues (2015:2) explain, "The geometric growth of the scientific corpus allows new opportunities for studying research practices with large-scale evidence and for testing empirically their effectiveness at producing the most reliable evidence." Although the scale of 
the new SoS was new, what really separated it from its predecessor was a willingness to transition from a strictly descriptive science to a diagnostic and experimental one. describing the frontier of the field, Fortunado et al. (2018:6) write, "Engaging in tighter partnerships with experimentalists, [SoS] will be able to better identify associations discovered from models and largescale data that have causal force to enrich their policy relevance." The statistical power of the new science and its willingness to experiment on science itself made it a perfect fit for science leaders and funders looking for evidence-based interventions for improving research integrity and quality.

Methodological and statistical criticism. Finally, prescient critiques can be traced back to the work of statisticians like Jacob Cohen and psychological methodologists like Paul Meehlspecifically their criticisms of null hypothesis significance testing. However, the replication crisis breathed new life into debates that seemed to flare up and burn out every couple of decades. Fiona Fiddler, a speaker at the Metascience Symposium described the despondency she felt when she finished her $\mathrm{PhD}$ which was a historical and philosophical analysis of the criticisms of p-values and null hypothesis testing across three fields: "When I finished that in 2005 I thought, 'This is going nowhere. I've just documented five, six decades of this going exactly, precisely nowhere. There's nothing left for me here.' Turns out I was wrong about that. It was going somewhere. And now we're all here in the middle of some kind of revolution." What was seen as a fastidious, marginal subfield became a hot topic as researchers looked for the causes of the replication problems. Within a couple of years, these critiques that had floated around for decades suddenly became objects of intense interest.

There is nothing intrinsic in these three trends that has forced them to converge. In fact, in many ways, these domains remain distinct. For instance, some open science activists are motivated primarily by the democratization of knowledge and have little interest in research integrity. And much research in the quantitative study of science is completely independent of issues of open science and research integrity. At the same time, there are shared goals that have encouraged these areas to find common cause (Figure 1).

FIGURE 1 ABOUT HERE 
Criticisms of scientific practice by methodologists have provided new arguments to make science more open. Scholars engaged in quantitative studies of science depend upon access to masses of data and, thus, are eager to encourage greater openness. And methodologists have embraced quantitative studies demonstrating statistical irregularities in entire fields.

Still, statistical debates and replication scandals have occurred before. What has given metascience its potency has been its ability to attract financial and intellectual investment and, then, use that investment to create self-perpetuating cycles of research, critique, and intervention. This is why when we later asked Fidler what happened to make her feel she was living through a revolution, she responded, "Brian Nosek happened." Brian Nosek has been a major interlocuter in debates about the reproducibility crisis. Yet, what this tongue-in-cheek comment meant to convey was that previous periods of scientific self-reflection passed into nothingness once the energy animating them began to subside. What Brian Nosek and other metascientists have done is to funnel the energy and funding pouring into metascience into institutions that will influence science policy long after the news cycle surrounding the crisis narrative fades.

\section{THE INSTITUTIONALIZATION OF METASCIENCE}

We asked Bruce Fetzer, president of the philanthropic organization that was the primary funder of the Metascience Symposium, what distinguished this moment from debates about problems in science that happened in the past. He replied, "What's unique about it right now is that there's one widespread admission that this is pervasive through all of the different disciplines." The scale of the problem motivated significant investments:

I think what's different, is that the idea was powerful enough and its time had come to where private foundations stepped up and provided the seed capital, so you had foundations probably putting up a collective of $\$ 20, \$ 30$ million, and then, all of a sudden, this thing got a huge amount of visibility because the universities got involved. In the midst of the replication crisis, both private and public science funders grew concerned that their investments were being wasted. To counter this, the funders sought scientific methods of evaluating and managing their portfolios.

The evaluation and management of science has always been a challenging problem (Shapin 2010). Scientists typically have far more expertise in their area of research than managers, funders, or institutional leaders. Moreover, the direction and speed of basic research 
cannot be plotted out in advance. Research paths often become clear only after several steps are taken and seeming mistakes can yield significant developments.

Management problems become acute during periods of scandal when it seems as though the flexibility built into the system was little more than poor oversight. This leads to questions about how much autonomy is really necessary. As one metascientific manifesto argues, "Given that academic science is a largely public institution funded by public money, it is surprising that there is so little transparency and accountability for the research process. Beyond the published reports, science operates as a 'trust me' model that would be seen as laughably quaint for ensuring responsibility and accountability in state or corporate governance" (Nosek et al. 2012:625).

The replication crisis spurred funders to look for an alternative to the "trust me" model. At the Metascience Symposium, the director of insight and analysis at the Wellcome Trust, Chonnettia Jones remarked, "Two trillion dollars are spent on research and development around the world. Two trillion dollars. And what's really remarkable about a two trillion-dollar enterprise is that we actually don't know how it works, what works, and how to make it work better." Emphasizing the value of the transition from a descriptive to an evaluative, diagnostic, and therapeutic science, she continued, "If we're going to do research on the scientific process, then why would we not want to use that evidence to be able to improve the research enterprise? So, not just doing meta-research for the sake of research, but for the purpose of actually translating that evidence, those insights into change that benefits everyone."

Unlike the already existing work on the production, communication, and dissemination of science, the new field promised the type of research that could be taken to a CEO or a Board of Directors as evidence for change. As Dawid Potgieter, Director of Programs in Discovery Science at the Templeton World Charity Foundation, told the Symposium audience, "I don't think there will be very much change unless we have this kind of research, this field of metascience because funders [,] we are very bureaucratic. We've very scared of messing things up. So, having some kind of solid evidence to make a change really is immensely valuable."

The natural conservatism of funders, both private and public, has led to investments in programs designed to promote accountancy and accountability. In a strategic move to alter the trajectory of science, Arnold Ventures provided major funding to create several metascientific institutes which were designed to monitor, diagnose, and improve scientific practice in an 
ongoing way. They provided seed money for the foundation of METRICS, founded by John Ioannidis, with the explicit goal to "connect the disparate elements of this field and enhance their synergy and collective efficiency towards the goal of improving published research" (Ioannidis et al. 2015:4).

The Center for Open Science (COS), also founded with seed money from Arnold Ventures, is a useful example of the systemic vision of metascience. Unlike many of the newly funded organizations which focus on specific aspects of open science or quantitative studies of science, the COS draws together all the facets of metascience into a coherent and integrated project. In a recent strategy statement (COS 2017), for instance, they detail their goals which include, first, producing metascience which provides the rationale for institutional change. Toward this end, they coordinated the first mass replication project (OSC 2015), which was hailed as one of the top scientific breakthroughs by Science. Their success has led to several other mass replication efforts including one aimed at replicating 50 studies in preclinical cancer biology. Second, the OSF creates open science infrastructure that will ensure that "scholarly content is preserved, connected, and versioned to foster discovery, accumulation of evidence, and respect for uncertainty." To further this goal, they, they created a new repository that can store data, code, and protocols and they host preprint servers for a number of fields including psychology, sociology, and engineering. Finally, they work to "nudge" funders and journals to improve their practice. The COS helped organize a group of researchers to write what became the TOP Guidelines (Nosek et al. 2015). These provided a framework for journals to sign on to that could help them diagnosis problems with and, hopefully, improve their transparency and openness. Currently, over 5,000 journals have signed onto the statement. The COS also intersects institutionally with other organizations in this metascientific space creating a growing network. For instance, they partner with Retraction Watch, hosting their retraction database, and built the open-source platform SHARE in partnership with the Association of Research Libraries, who have led discussions on open access.

\section{FROM MORAL ENTREPRENEURS TO ENGINEERS OF EFFICIENCY}

The warrant for metascience is largely founded in the replication crisis. Metascience is framed as both an important diagnostic tool and the treatment. Or, as the strategy statement from the Center for Open Science (COS 2017) puts it, their metascientific projects (including their 
much-lauded mass replications) are about "acquiring evidence to encourage change" which they then support through an "ecosystem" of technological and training services. Empirical evidence of low replication rates in a field, for instance, can stoke a narrative of "replication crisis" and motivate funders, journals, and institutions to perform further replications, establish data deposition standards, or develop training in data management, all services metascientific organizations perform.

Metascientists seem happy to position themselves in a place to benefit from anxieties about a reproducibility crisis, yet in both their interviews and published manifestos their attentions extend beyond the empirical issues in any specific field, to fundamental questions about the organization of science. For instance, Jonathan Schooler, one of the organizers of the Metascience Symposium published an article entitled "Metascience could rescue the 'replication crisis" (2014). Yet, when we asked him about it, he told us he regretted using the crisis language explaining that "the word crisis is probably overblown." When we asked Brian Nosek if he thought science was in the midst of a reproducibility crisis, he said, "Is there a crisis? I think there has been and maybe continues to be a crisis of confidence. In the sense that that's almost obviously true. There is a massive amount of increased attention to this, consternation about it, arguing about it. So, if that's what makes a crisis, then, yeah, sure, we're in a crisis."

Metascientific writings show a similar trend. For instance, in their manifestos and programmatic articles (Begley and Ioannidis 2014; Ioannidis et al. 2015; Munafò et al. 2017; Nosek \& Bar-Anan 2012; Nosek et al. 2012), the word "crisis" hardly appears. One article mentions the crisis, suggesting that it is "debatable." This same article, however, immediately pivots immediately what is a unifying thread for metascientists. Munafò et al. (2017:1) note that metascience "is flourishing and has generated substantial empirical evidence for the existence and prevalence of threats to efficiency in knowledge accumulation."

In their papers, talks, and interviews, the language of "efficiency" is ever-present. For instance, in the articles mentioned above, Begley and Ioannidis (2014) frame their entire argument around the balance between "efficiency" and "waste" in research, and both "Scientific Utopia I" and "II" discuss efficiency in their abstracts. As Open Knowledge Foundation's Jarmo Eskelinen told us, "transparency is nice, and it's been sort of the low-hanging fruit" but admitted that "the primary goal is efficiency." 
At first blush, this may seem like an odd choice. "Efficiency" does not produce the same feelings of urgency that "crisis" sparks, yet it carries with it some significant advantages organizationally. Most generally, crises pass. Earthquakes stop. Tsunamis reach their crest and ebb. The same can be expected for the replication crisis. As Becker notes, moral crusaders often find themselves out of a job once reforms have been enacted. What the language of efficiency allows metascience to do is to transition to a different type of relationship with the rest of science, one that is more stable and sustainable.

\section{SCIENCE AS THE PRODUCTION, CURATION, AND MAINTENANCE OF INFORMATION}

The blueprint is a familiar one for our information age - use advances in technology to innovate and transform stagnant industries. Efficiency in basic science is a complex issue (which we will explore later). What metascientists mean, however, by efficiency in the context of scientific research involves removing the "friction" that slows down scientific communication and research. Because it requires the transformative introduction of new technologies into stagnant fields, efficiency can be achieved by what technologists mean by "disruption." This is why, sprinkled throughout this literature, there are references to various cutting-edge social systems based on behavioral economic ideas like crowd-sourcing (Uhlmann et al. 2019) and decision markets (Munafò et al. 2015) as well as Web 2.0 companies like Spotify (Ross-Hellauer et al. 2019) and Reddit (Nosek \& Bar-Anan 2012). The replication crisis may have led us off the path of good science, but the way forward is innovation, not a reversal.

At the core of this disruption is the transition from the physical world to the digital. As open data advocate Rufus Pollock explained, "We've moved from atoms to bits. That shift has never happened before in human history and bits are different. They're costlessly copyable. Wow. The miracle of the loaves and fishes in the Bible. Oh my God." What this means for science is that there need be no restrictions on access to data. At one time, sharing data meant creating physical copies of things like spreadsheets. It was time-intensive and expensive. The shift to digital storage and instant communication has removed those barriers and has opened up new possibilities for information sharing and collaboration.

This has important implications for those tasked with addressing the replication crisis. One of the speakers at the Metascience Symposium explained, 
We talk a lot about science being self-correcting and that mechanism, whatever that mechanism was, being broken at the moment. And, open science is one part of how to fix that. So, you can only properly evaluate and scrutinize someone's work, someone's research, if you have access to all of the pieces, to the code, to the raw data. If the goal is to create a culture in which self-correction functions efficiently, or how we think it should, or better than it is now, or whatever, then making it open seems like it has to be the first step to that.

If there is little or no cost associated with copying, sharing, and hosting data, that provides a means for ongoing self-correction. While most scientists would endorse the classical Mertonian picture that science operated based upon "organized skepticism" based upon the "communalism" of the data, the digital revolution provides a means to take these loosely defined norms and bake them into the very infrastructure of science. The norm of communalism becomes a demand from the journal or funder to deposit data into an open repository and, thus, organized skepticism becomes possible on a hitherto unknown scale.

Yet, the potential benefits of utilizing new systems of scientific communication and data storage extends far beyond merely providing better accounting. As Eloy Rodrigues, board member of COAR, told us, "the current system is really still very tied with the pre-digital technologies and ways of doing things. We are not really using all the possibilities of the web and now probably in the future of data mining and artificial intelligence and things like that." Similarly, SPARC executive director Heather Joseph explained the downsides to the current fragmented system of data access: "It essentially prevents science from proceeding seamlessly, right? If you can only work on certain pieces in isolated chunks and you can't use computational technology, you can't use machine learning or AI, you can't go across a full corpus seamlessly in the online environment, science gets slowed down."

Metascientists arguing that moving beyond the "pre-digital," paper-based system of science and into a network of open access repositories with interoperable data can propel science into a new future in which all scientific fields intersect with data science. It is in this imagined future that the three strands of metascience come together. Statistical methodologists achieve transparency and, with it, a better chance at self-policing. Open science activists achieve a free flow of information. And data scientists achieve new and growing datasets that are the grist for their work. 
This metascientific philosophy of science, in which scientists are in the business of producing, curating, and maintaining data differs markedly from philosophies of science which revolve around the theory or the experimental system. It is a post-human theory of science in which the limitations of human bias can only be overcome with total transparency and machine intervention. This vision of science aligns with existing trends in multiple fields which have seen an influx of data and computer scientists. The field of genomics, for instance, has already become deeply interwoven with data science (Arribas-Ayllon et al. 2019).

Metascience has utilized the energy from the crisis narrative to build infrastructure and draw several existing trends into a mutually beneficial alignment. However, as it continues to grow and seeks to make further changes in scientific practice, metascience confronts daunting challenges. Respondents brought up several. There are significant funding challenges to creating ongoing mass replication efforts and curating ever-growing repositories. Getting buy-in from disciplinary scientists to actually produce reusable data is difficult. But a more significant issue strikes at the heart of the metascientific enterprise - navigating the relationship between metascience and disciplinary science.

\section{WHAT IS META ABOUT METASCIENCE?}

Sciences can, somewhat simplistically, be defined as professional groups with authority over a particular domain of reality. Under this definition, the domain of authority claimed by metascientists is scientific practice itself. This may not, at present, include things like actual experimental practice — filling pipettes or running particle accelerators — but it encompasses many activities that were typically negotiated within specific fields. Norms of evidence collection, analysis, data storage, reporting standards, and scientific communication are all within the ambit of current metascientific project. And there are indications that metascientists plan even more ambitious interventions into currently autonomous areas of science. ${ }^{6}$

\footnotetext{
6 At the Metascience Symposium, DARPA program manager Adam Russell explained that his agency was tasked with synthesizing research to provide guidance for military and political decisions. However, reproducibility problems and the general low quality of scientific research, especially in the social sciences, resulted in a deep skepticism of the published literature. To counter that, the agency was funding a spate of metascience projects designed to improve the science through a combination of open science initiatives and machine learning.

"Systematizing Confidence in Open Research and Evidence" is a \$7.6 million investment in trying to create an automated system to evaluate
} 
Metascience poses a deep dilemma. On one hand, they are engaged in a seemingly commonsensical and uncontestable mission to make science more transparent and efficient. They attempt to formalize the loosely defined "norms" of science. Nosek explained that the Center for Open Science was

the practical application of our substantive research interest, which is the gap between scientific values and scientific practices. How we idealize science to operate, what we want it to do, versus how it actually operates in daily life. And what we have the opportunity to do organizationally is address these dual challenges. The structure, the system, the culture that's been created, and how that structure and system leverages all of these biases down in the individual level that end up creating bias in the system, end up hurting the pace of discovery.

Removing bias and hastening discovery, bridging the gap between our ideals and practices - that all sounds great. And, for those who claim to defend science's unique claim to epistemic authority, how could they argue against turning the tools of science back on the scientists themselves to achieve these laudable goals? From what epistemic authority could one launch such a challenge? Who can reject calls for transparency? What argument can be made against efficiency?

On the other hand, the project itself seems to be founded on a set of ideas that find few supporters in the philosophy or social studies of science: that "science" is a coherent entity on which to intervene, that there is a singular method for science, that "efficiency" is a meaningful concept in the area of basic research. The fundamental stance of metascience would seem to be at odds with one of the central findings of science studies, that "science is not one, indivisible, and unified, but that the sciences are many, diverse, and disunified." (Shapin 2010:5). Rather than a unified set of practices, empirical researchers have found an incongruous mixture of

\footnotetext{
behavioral and social science research. "Big Mechanism" is comprised of $\$ 45$ million in funding and seeks to use machine learning to read the academic literature in cancer and develop novel causal models. And, "Automating Scientific Knowledge Extraction" is a \$1 million bet on using artificial intelligence to automatically collect information, extract its useful components, integrate this information into evolving models with the goal of eventually "generating [...] machine-generated hypotheses."

These projects make it clear that the ultimate goal, for at least some metascientists, is not merely the improvement of existing disciplines, but a radical transformation in which disciplinary science feeds into evolving artificial intelligence systems which evaluate, synthesize, and even conduct research.
} 
practices, methods, and standards which has led the field to largely embrace the image of science as a disunified amalgam of distinct epistemic cultures (Galison \& Stump 1996; Knorr Cetina 1999).

The sorts of qualitative differences discovered through historical and case studies present a challenge to a field with designs on measuring and manipulating capital-S science and whose whole claim to authority rests on the coherence of domain of science. It is not necessary to read between the lines to glimpse the scale of the ambition: "Unfortunately, there is no centralized means of aligning individual and communal incentives via universal scientific policies and procedures" (Nosek et al. 2015:1423). Metascience is only rational in a world where science can be unified by "universal policies and procedures" and, thus, where it makes sense to think about science as a unitary object. That alone makes their measurements and evaluations meaningful and makes interventions logical. The unity of science and metascience emerge together. Because of this, however, the difficulties in uncovering the universal aspects of science are recapitulated in metascience.

\section{THE FRAGILE UNITY OF METASCIENCE}

Modern metascience is characterized by a disengagement with the theoretical and philosophical complexities that have emerged in the past half century of philosophy and social studies of science. Such strategies are common in fields where empirical work is in constant danger of getting derailed by theoretical disagreements (Panofsky 2014; Peterson 2018). The kind of ongoing, seemingly irresolvable theoretical debates in science studies was clearly unattractive to some metascientists. We asked Jonathan Schooler, one of the organizers of the Metascience Symposium, what distinguished this iteration of metascience from the many philosophies and empirical studies of science that preceded it. He argued,

A lot of discussion about science and scientific processes was very qualitative, was very descriptive. I mean, the Kuhnian paradigm, it's brilliant ideas, but how do we translate that into a testable, empirical predictions? I think that increased appreciation of the way in which different metascientific questions can be addressed empirically and the establishment of quantifiable paradigms to test different aspects of it is definitely consolidating. 
Interviewer: When you think about the normative side to it, what are the values or what are the norms that meta-scientists are championing?

Schooler: Well, I mean, I think that the number one norm is really a shift to finding ingenious ways of using the scientific method and, in particular, quantification, to draw insights about the scientific process. That was one of the things that really thrilled me about the metascience meeting was just all these different- oftentimes, just really ingenious- approaches that the researchers were finding to get at these processes. Less armchair, less qualitative distillation of impressions and more hard-nosed empiricism. The desire for "hard-nosed empiricism" meant that no qualitative studies of science were presented at the Metascience Symposium. There was a session which several respondents called the "skeptics panel," but the critiques were focused on technical issues in metascience rather than the theoretical challenges that are common features of debates in STS. Jan Walleczek, Director of Research at the Franklin Fetzer Fund, helped organize the conference. He told us that avoiding these sorts of theoretical debates was a key decision regarding invitations to the symposium. There were discussions early on regarding whether to invite a science studies figure like Bruno Latour, but it was decided that this first event should occur with as much theoretical unity as possible.

This was effective. One of the attendees at the conference noted that she has attended a "weird conference" about similar issues that was attended by science studies scholars as well as experimental scientists. She lamented the unproductive nature of the conference because they could not agree on even basic principles. In contrast, she explained that the Metascience Symposium "was probably the first where I talked about metascience in such a constructive way."

I think the way that the Metascience Symposium in Stanford was focused, it included people who looked at this or worked according to the same empirical cycle as each other, so you form a theory, you derive a hypothesis, you gather data, you test your prediction, you evaluate your findings. That kind of logic. And in the other conference, where I was with all these different disciplines, that was already not agreed on, and whether there is one truth, or whether that is subjective. Yeah, if you can't agree about these things, then it's really hard to come up with solutions about what will make science better, because you don't have a same idea of what would a better science look like. 
METASCIENCE

The broad consensus of modern metascience — quantitative, atheoretical, positivist—provides the framework for "doable problems" (Fujimura 1992). And the lack of reflexivity provides the courage to intervene on science as a whole.

\section{METASCIENCE AND THE AUTONOMY OF DISCIPLINES}

Bourdieu (1994) has written that the defining feature of scientific fields is that the primary consumers and evaluators of science are other scientists. Whereas a field like art produces for a market of collectors and critics, scientists produce for each other. A similar idea is captured in Collins' (1981) concept of "core-set," which is the collection of scientists that are legitimately able to contribute to a scientific area. Both these concepts emphasize autonomy. The idea of science as a social enterprise, floating above and detached from the murkiness of politics and confusions of culture, accountable only to itself, is not seriously defended after scholarship on the AIDS Crisis, global warming, and the genetics of race. Yet, there is a kernel of truth to this vision. In many areas of science, researchers primarily produce research for other researchers. Scientific communities are the producers, primary audience, and evaluators of science.

Metascience suggests a world in which scientific products have another important consumer and evaluator. The immunologist, the neuropathologist no longer produces work just for immunologists and neuropathologists. Their work is absorbed into metascience and becomes the basis for measurements of their statistical features, level of openness, and degree of efficiency. These measurements get compared across fields or across the same field over time in evaluations. These evaluations, in turn, become the motivation for new interventions.

It is not surprising that this can create tensions between metascience and the disciplines it overlooks. Jon Yewdell, Chief of the Cellular Biology Section of the NIH, was invited to the "skeptics panel" at the Metascience Symposium because of his criticisms of the crisis narrative. In an interview, he explained how the popularized accounts of the crisis in science produced political pressure which, in turn, led to greater demands for transparency and accountability, demands he left were, ultimately, unproductive. Having attended the symposium and read, he left thinking, "These are really smart people, brilliant in their abstract ability to think but, Jesus, you got to be in the trench to see how it really works and how slippery the truth really is." Later, he 
lamented the lack of communication between metascientists and disciplinary researchers: "They don't seem to be terribly interested in feedback from us."

Individually, metascientists are more cautious and modest in their approach. For instance, Daniele Fanelli, another presenter at the symposium and a metascientist who published one of the earliest meta-analytic articles on research integrity (Fanelli 2009), has become much more critical of the reformist strand of the field. After a decade in the field, he said he has come to believe that "Every actual science realized is going to depart to a greater or lesser extent from some ideal modus operandi, which is pretty much never realized. And there are reasons why these practices depart. And at least in part these reasons are not just deleterious, they are actually ways in which people are able to compensate for what otherwise would be insurmountable limitations." Jevin West, another presenter, told us "There was a time when I really did believe truly in a Swiss army knife metaphor for some of these methods [...] But the more qualitative work I do, the more I realize we have to be careful about not relying too much on some of these macro level tools." Moreover, several interviewees expressed a desire for greater communication between metascience and more historically, philosophically, or qualitative traditions.

However, to the degree that metascience is able to attract research funding and public attention through the use of impressive looking quantitative analyses and mass replications, there seems to be little reason for the field to open itself to the types of philosophical quagmires that exist in the philosophy and social studies of science. More problematically, as metascience institutionalizes - as it creates self-perpetuating institutes, conferences, journals, and departments - it creates the potential for an ongoing gulf between metascience and disciplinary science as metascientists no longer come to metascience through a discipline, but are raised intellectually in the heady mixture of big data, open science, statistical mechanics, and little knowledge how bench science is done. Ironically, despite ostensibly pursuing a philosophy of unified science, metascience ends up further fragmenting science by appropriating portions of scientific authority. This creates new forms of expertise, practice, and scientific capital. It also produces new forms of surveillance and accountability demands.

\section{CONCLUSION}

Metascience has arisen at the same time as both the replication crisis and anxieties over post truth politics. Its promise rests on being able to solve these problems by investing more 
heavily in modernist ideals. Bigger science, greater transparency, more accountability-these are the mottos of the movement. This has been a successful pitch to funders looking to maximize their investments and make frontier science more administratively legible. As a scientific social movement, it has been unusually successful and influential.

Yet, the project raises some significant questions for the sociology of science. Bourdieu once wrote

The official vision of science is a collective hypocrisy capable of guaranteeing the minimum of common belief that is necessary for the functioning of social order; the other face of science is both universally known to all those to take part in the game and unanimously disguised, as a jealously guarded 'open secret' (economists would call it 'common knowledge'). Everyone knows the truth about scientific practices, which the new sociologists of science noisily discover and unveil." (2004:77)

Bourdieu dismissed STS for its obviousness. Everybody knows that lab science and published science look nothing alike, that all the ambiguity and tacit knowledge and social negotiation disappear as claims leave the lab.

A segment of scientists, however, attacked STS research from a very different perspective. They argued it amounted to little more than charlatanism and postmodern relativity. The "Science Wars" were, in a sense, a way for scientists to emphatically reject any claim on science made by social scientists and humanities scholars (Gross \& Levitt 1997; Sokal \& Bricmont 1999).

If STS did little more than discover an "open secret," metascience treats those same practices as an active scandal. Metascience exploits the "collective hypocrisy" of science because it directly challenges, as Nosek noted, the differences between scientific ideals and scientific practice. Moreover, it presents a different, more direct challenge to the autonomy of science than STS ever did because it comes dressed in a white lab coat and speaks in quantitative data. Because the entire conversation is happening in the commonsensical language of the Science Warrior, there is no epistemic location from which to reference history or interpretation or tacit knowledge. Everything is either data than can be reabsorbed into the metascience project or it is nothing. Because it lacks the nuance that STS has developed, everything that falls short of the ideals is made to look fraudulent or, at best, problematic. 
Yet, drawing on the STS research that metascience has almost entirely sidestepped suggests that there are three poles, a triangle into which any individual scientific practice can be placed and interpreted. There are its rhetorical ideals, unquestionably poor practices, and practices that, while not in line with dominant rhetoric about the scientific method, are helpful or essential for a scientific community. This analytical distinction is fuzzy in practice but, as Harry Collins (2019) recently quipped, the distinction between the pond and the field may be muddy, but you can still drown in the pond. And, in this case, there are multiple ways to drownunethical/inefficient scientific practice and rash interventions that have unintended consequences.

Scientists are typically beholden to other scientists, but, as a community, share tremendous freedom over their own activities. Metascience enfolds many of these activities into its project, translating them into objects for enumeration, measurement, comparison, evaluation, and, ultimately, manipulation. As metascience institutionalizes and seeks reforms, their domain of authority increasingly abuts, and sometimes collides into, existing scientific authority. If the label "science" is a loose umbrella that covers a set of incongruous types of practice, then any attempt to diagnose and treat big-S Science is going to have uneven benefits or, even, cause new harms. The danger is exacerbated as reforms become system-wide. If metascience achieves its vision of becoming the diagnostician and therapist for science writ large, the "sciences" which have evolved in a thousand independent histories buffeted by internal and external forces are going to be increasingly treated as "Science."

The urge to understand and improve science is an understandable goal. In our current moment, when even basic, widely agreed upon facts are not sacrosanct, it seems urgent and necessary. Will metascientific reforms lead us out of the replication crisis? Will they deliver us from our epistemic crisis? These questions cannot be answered. But they suggest others, equally necessary and urgent: What is a replication crisis? What is the relationship of transparency to trust? How can we measure efficiency when basic science often does not have a known objective? Metascience has been a frantically productive empirical field devoid of theory. A final question: how much longer can that last? 


\section{REFERENCES}

Arribas-Ayllon, Michael, Andrew Bartlett, Jamie Lewis, Andrew Bartlett, and Jamie Lewis. 2019. Psychiatric Genetics : From Hereditary Madness to Big Biology. Routledge.

Baker, Monya. 2016. “Is There a Reproducibility Crisis?” Nature (533):452-54.

Becker, Howard S. 2008. Outsiders. Simon and Schuster.

Begley C. Glenn, and Ioannidis John P.A. 2015. "Reproducibility in Science.” Circulation Research 116(1):116-26.

Begley, C. Glenn, and Lee M. Ellis. 2012. "Drug Development: Raise Standards for Preclinical Cancer Research.” Nature 483(7391):531-33.

Belluz, Julia. 2017. “Cancer Scientists are Having Trouble Replicating Groundbreaking Research." Vox, Jan. 23. Retrieved on 6/25/20 from https://www.vox.com/science-andhealth/2017/1/23/14324326/replication-science-is-hard

Bohlin, Ingemar. 2004. "Communication Regimes in Competition: The Current Transition in Scholarly Communication Seen Through the Lens of the Sociology of Technology." Social Studies of Science, 34(3), 356-391.

Bourdieu, Pierre. 2004. Science of Science and Reflexivity. Polity.

Brown, Phil, Stephen Zavestoski, Sabrina McCormick, Brian Mayer, Rachel Morello-Frosch, and Rebecca Gasior Altman. 2004. "Embodied Health Movements: New Approaches to Social Movements in Health." Sociology of Health \& Illness 26(1):50-80.

Bunge, Mario. 1959. Metascientific Inquiries. C. C. Thomas.

Chivers, Tom. 2017. "These People are Trying to Fix a Huge Problem in Science.” Buzzfeed News, Jul. 29. Retrieved on 6/25/2020 from https://www.buzzfeed.com/tomchivers/signifying-nothing

Collins, Harry M. 1981. "The Place of the Core-Set in Modern Science: Social Contingency with Methodological Propriety." History of Science 19(1):6-19.

Collins, Harry M. 2019. Forms of Life: The Method and Meaning of Sociology. MIT Press.

COS. 2017. “Center for Open Science: Strategic Plan.” Retrieved on 6/28/20 from https://osf.io/x2w9h/

Crow, Raym. 2002. "The Case for Institutional Repositories: A SPARC Position Paper.” ARL Bimonthly Report, 223.

Daston, Lorraine, and Peter Galison. 2010. Objectivity. New York, NY: Zone Books. 
Epstein, Steven. 2008. Inclusion: The Politics of Difference in Medical Research. Chicago, IL: University of Chicago Press.

Eyal, Gil. 2019. The Crisis of Expertise. Polity.

Fieldler, Klaus, \& Schwarz, Norbert. 2016. "Questionable Research Practices Revisited.” Social Psychological and Personality Science, 7(1), 45-52.

Fanelli, Daniele. 2009. "How many scientists fabricate and falisify research? A systematic review and meta-analyses of survey data." PloS One, 4(5), e5738.

Fortunato, Santo, Carl T. Bergstrom, Katy Börner, James A. Evans, Dirk Helbing, Staša Milojević, Alexander M. Petersen, Filippo Radicchi, Roberta Sinatra, Brian Uzzi, Alessandro Vespignani, Ludo Waltman, Dashun Wang, and Albert-László Barabási. 2018. "Science of Science." Science 359(6379):eaao0185.

Freese, Jeremy, and David Peterson. 2018. "The Emergence of Statistical Objectivity: Changing Ideas of Epistemic Vice and Virtue in Science.” Sociological Theory 36(3):289-313.

Frickel, Scott, and Neil Gross. 2005. "A General Theory of Scientific/Intellectual Movements." American Sociological Review 70(2):204-32.

Fujimura, Joan H. 1992. "Crafting Science: Standardized Packages, Boundary Objects, and 'Translation.'” Pp. 168-211 in Science as Practice and Culture, edited by A. Pickering. Chicago, IL: University of Chicago Press.

Galison, Peter Louis, and David J. Stump (Eds.). 1996. The Disunity of Science: Boundaries, Contexts, and Power. Stanford University Press.

Gardner, Howard. 1987. The Mind's New Science: A History of the Cognitive Revolution. Basic books.

Gelman, Andrew. 2010. "The Experiments are Fascinating. But Nobody can Repeat Them." New York Times, Nov. 19. Retrieved 6/25/20 from https://www.nytimes.com/2018/11/19/science/science-research-fraudreproducibility.html? searchResultPosition $=1$

Gelman, Andrew. 2016. "What has happened down here is the winds have changed." Statistical Modeling, Causal Inference, and Social Science. Retrieved on 5/29/20 from https://statmodeling.stat.columbia.edu/2016/09/21/what-has-happened-down-here-is-thewinds-have-changed/ 
Gross, Paul R., \& Levitt, Norman. 1997. Higher Superstition: The Academic Left and Its Quarrels with Science. Johns Hopkins University Press.

Harris, Richard. 2017. Rigor Mortis: How Sloppy Science Creates Worthless Cures, Crushes Hope, and Wastes Billions. Basic Books.

Ioannidis, John P. A. 2005. "Why Most Published Research Findings Are False." PLOS Medicine 2(8):e124.

Ioannidis, John P. A., Daniele Fanelli, Debbie Drake Dunne, and Steven N. Goodman. 2015. "Meta-Research: Evaluation and Improvement of Research Methods and Practices." PLOS Biology 13(10):e1002264.

John, Leslie K., Loewenstein, George, \& Prelec, Drazen. 2012. "Measuring the Prevalence of Questionable Research Practices with Incentives for Truth Telling." Psychological Science, 32(5), 524-532.

Kelty, Chris. 2008. Two Bits: The Cultural Significance of Free Software. Duke University Press.

Knorr Cetina, Karin. 1999. Epistemic Cultures: How the Sciences Make Knowledge. Cambridge, MA: Harvard University Press.

Leonelli, Savina. 2016. Data-centric Biology: A Philosophical Study. University of Chicago Press.

Mallapaty, Smriti. 2020. "Popular Preprint Servers Face Closure Because of Money Troubles." Nature 578, 349.

McCormick, Sabrina. 2007. "Democratizing Science Movements A New Framework for Mobilization and Contestation." Social Studies of Science 37(4):609-23.

Merton, Robert K. 1973. The Sociology of Science: Theoretical and Empirical Investigations. University of Chicago press.

Miller, George A. 2003. "The Cognitive Revolution: A Historical Perspective." Trends in Cognitive Sciences 7(3):141-44.

Mirowski, Philip. 2018. "The Future(s) of Open Science.” Social Studies of Science 48(2):171203.

Morris, Charles. 1946. "The Significance of the Unity of Science Movement." Philosophy and Phenomenological Research 6(4):508-15. 
Munafò, Marcus R., Brian A. Nosek, Dorothy V. M. Bishop, Katherine S. Button, Christopher D. Chambers, Nathalie Percie du Sert, Uri Simonsohn, Eric-Jan Wagenmakers, Jennifer J. Ware, and John P. A. Ioannidis. 2017. “A Manifesto for Reproducible Science.” Nature Human Behaviour 1(1):0021.

Munafo Marcus R., Pfeiffer, Thomas., Altmejd, Adam, Heikensten, Emma, Almenberg, Johan, Bird, Alexander, Chen, Yiling, Wilson, Brad, Johannesson, Magnus, \& Dreber, Anna. 2015. "Using Prediction Markets to Forecast Research Rvaluations.” Royal Society: Open Science.2: 150287.

Nelson, Nicole C. 2020. “The Methodologists: A Unique Category of Scientific Actors.” Engaging Science, Technology, and Society 6(0):20-33.

Nosek, B. A., G. Alter, G. C. Banks, D. Borsboom, S. D. Bowman, S. J. Breckler, S. Buck, C. D. Chambers, G. Chin, G. Christensen, M. Contestabile, A. Dafoe, E. Eich, J. Freese, R. Glennerster, D. Goroff, D. P. Green, B. Hesse, M. Humphreys, J. Ishiyama, D. Karlan, A. Kraut, A. Lupia, P. Mabry, T. Madon, N. Malhotra, E. Mayo-Wilson, M. McNutt, E. Miguel, E. Levy Paluck, U. Simonsohn, C. Soderberg, B. A. Spellman, J. Turitto, G. VandenBos, S. Vazire, E. J. Wagenmakers, R. Wilson, and T. Yarkoni. 2015. "Promoting an Open Research Culture.” Science 348(6242):1422-25.

Nosek, Brian A., and Yoav Bar-Anan. 2012. "Scientific Utopia: I. Opening Scientific Communication." Psychological Inquiry 23(3):217-43.

Nosek, Brian A., Jeffrey R. Spies, and Matt Motyl. 2012. “Scientific Utopia: II. Restructuring Incentives and Practices to Promote Truth Over Publishability.” Perspectives on Psychological Science 7(6):615-31.

Open Science Collaboration. 2015. "Estimating the Reproducibility of Psychological Science." Science 349(6251).

Panofsky, Aaron. 2014. Misbehaving Science: Controversy and the Development of Behavior Genetics. University of Chicago Press.

Peterson, David. 2017. "The Depth of Fields: Managing Focus in the Epistemic Subcultures of Mind and Brain Science.” Social Studies of Science 47(1):53-74.

Prinz, Florian, Thomas Schlange, and Khusru Asadullah. 2011. "Believe It or Not: How Much Can We Rely on Published Data on Potential Drug Targets?” Nat Rev Drug Discov 10(9):712-712. 
Ross-Hellauer, Tony, Fecher, Benedikt, Shearer, Kathleen, \& Rodrigues, Eloy. 2019. "Pubfair: A Framework for Sustainable, Distributed, Open Science Publishing Services.” White paper, version 1.

Schooler, Jonathan W. 2014. "Metascience Could Rescue the 'Replication Crisis." Nature, 515, 9.

Shapin, Steven. 2010. Never Pure: Historical Studies of Science as If It Was Produced by People with Bodies, Situated in Time, Space, Culture, and Society, and Struggling for Credibility and Authority. JHU Press.

Sokol, Alan, \& Bricmont, Jean. 1999. Fashionable Nonsense: Postmodern Intellectual's Abuse of Science. Picador.

Smaldino, Paul E., \& McElreath, Richard. 2016. "The Natural Selection of Bad Science.” The Royal Society: Open Science, 3, 160384.

The Advisory Council of EarthArXiv. 2020. "EarthArXiv response regarding COS's new funding model." Accessed on 5/25/2020 from https://eartharxiv.github.io/cos.html

Uhlmann, Eric Luis, Charles R. Ebersole, Christopher R. Chartier, Timothy M. Errington, Mallory C. Kidwell, Calvin K. Lai, Randy J. McCarthy, Amy Riegelman, Raphael Silberzahn, and Brian A. Nosek. 2019. "Scientific Utopia III: Crowdsourcing Science." Perspectives on Psychological Science 14(5):711-33.

Yong, Ed. 2016. "The Inevitable Evolution of Bad Science: A Simulation Shows how the Incentives of Modern Academia Naturally Select for Weaker and Less Reliable Results." The Atlantic, Sept. 21. Retrieved 6/25/20 from https://www.theatlantic.com/science/archive/2016/09/the-inevitable-evolution-of-badscience/500609/ 


\section{FIGURES}

Figure 1: The Strands of Metascience

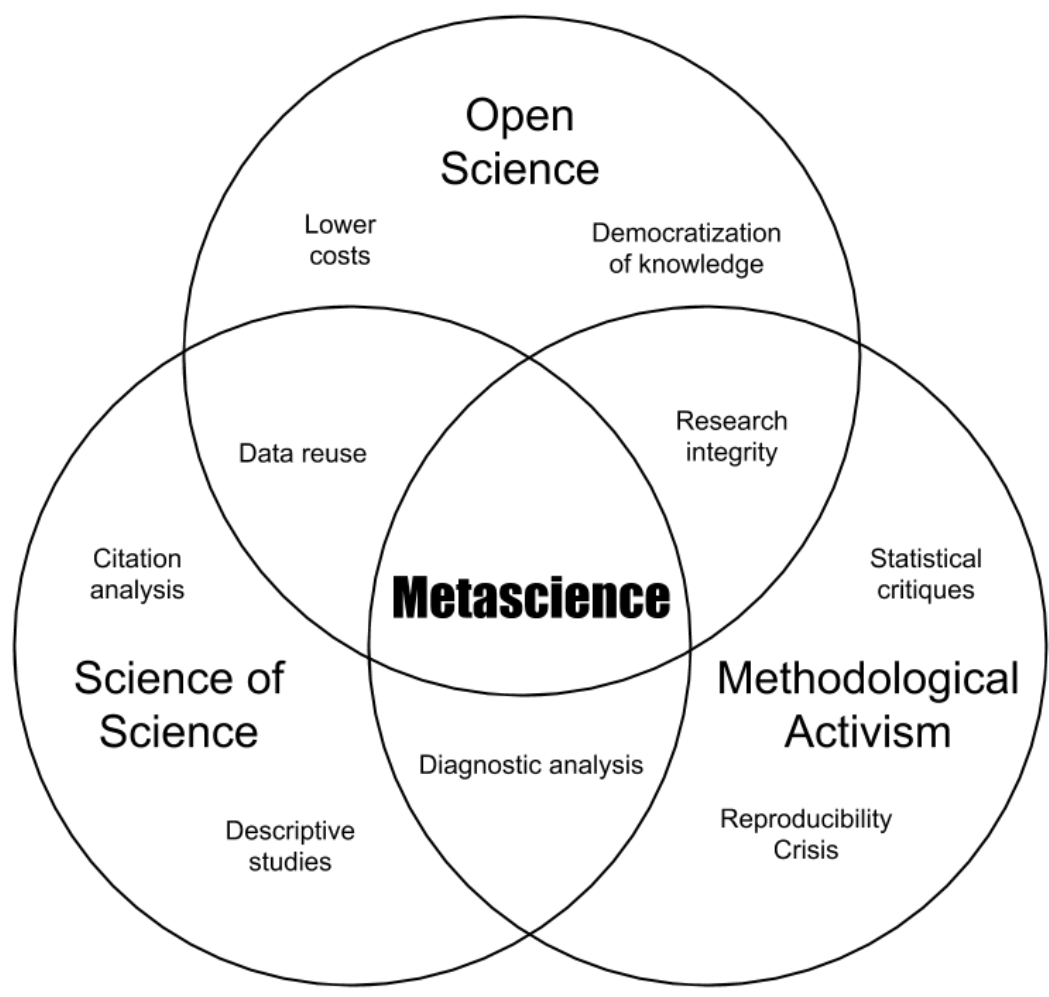

